\title{
THE ROLE OF MATRIX TABLET IN DRUG DELIVERY SYSTEM
}

\author{
NITA MONDAL ${ }^{*}$ \\ Pulla Reddy Institute of Pharmacy, Near Dundigal Air Force Academy, Annaram (V), Jinnara (M), Telengana \\ Email: nitamondal@gmail.com \\ Received: 11 Aug 2017, Revised and Accepted: 28 Nov 2017
}

\begin{abstract}
Matrix tablet is an important tool for controlled and sustained release dosage forms. The oral route remains the most common route for the administration of drugs. Tablets offer the lowest cost approach to sustained and controlled release dosage forms. The hydrophilic polymer matrix is widely used in this dosage form. The use of different polymers in controlling the release of drugs has become the most important tool in the formulation of matrix tablets. The drug releases by both dissolution-controlled as well as diffusion-controlled mechanisms from the matrix. The development of oral controlled release systems has been a challenge to formulation scientists due to their inability to restrain and localize the system at targeted areas of the gastrointestinal tract. There are several advantages of matrix devices including improved patient compliance due to less frequent drug administration, reduction of fluctuation in steady-state drug levels, maximum utilization of the drug, increased safety margin of a potent drug. This review aims on the discussion of different materials used to prepare matrix tablets, different types of matrix tablets and the drug release mechanism from the matrices.
\end{abstract}

Keywords: Sustained release, Controlled release, Diffusion, Erosion and matrix

C 2018 The Authors. Published by Innovare Academic Sciences Pvt Ltd. This is an open access article under the CC BY license (http://creativecommons.org/licenses/by/4.0/) DOI: http://dx.doi.org/10.22159/ijap.2018v10i1.21935

\section{INTRODUCTION}

Historically, the oral route is considered as a most popular route in the administration of the drug. This is because of the fact the gastrointestinal physiology offers more flexibility in designing dosage form than any other route. Approximately $50 \%$ of the drug products available in the market are administered orally. Tablets are the most commonly and widely used dosage form. This type of drug delivery system is called conventional drug delivery system and is known to provide an immediate release of the drug. Such immediate release products result in relatively rapid drug absorption and the onset of accompanying pharmacodynamics effects [1-3].

Developing safe and efficient drug delivery system is one of the major challenges in the pharmaceutical industry. Therefore, the characteristics of drugs as well as the form in which they are delivered must be optimized. Among the oral products, matrix tablets are an important tool for the controlled and extended release of drug. The oral sustained release system is the most popular, desirable and preferred method of administrating therapeutic agents for systemic effects in order to increase patient compliance and the therapeutic efficacy. The tablet matrix is formed by using both hydrophilic polymers and hydrophobic lipids to prolong and sustain the rate of drug release. Currently, much attention is being paid on the development of matrix sustained formulations such as matrix tablets containing hydrogels [4].

The matrix system containing hydrophobic lipid also has been widely used in controlled drug delivery applications due to their chemical inertness, cost-effectiveness, regulatory acceptance and flexibility of achieving the desired drug release profile [5, 6].

Because of increased complications and expense involved in the marketing of new drug entities, greater attention has focused on the development of modified release drug delivery system. A controlled release matrix system delivers the drug locally or systemically at a predetermined rate for a specified period of time $[7,8]$. The goal of such systems is to provide desirable delivery profiles that can achieve therapeutic plasma levels [9]. Drug release is dependent on polymer properties, thus the application of these properties can produce well characterized and reproducible dosage forms. Sustained release systems include any drug delivery system that achieves slow release of drug over an extended period of time. If the system is successful in maintaining constant drug levels in the blood or target tissue, it is considered as a controlled-release system.
Controlled release systems can be influenced by physiological conditions such as motility, ions, $\mathrm{pH}$ and enzymes. Hydrophilic matrix systems are among the most commonly used means for oral controlled drug delivery as they can reproduce a desirable drug profile and are cost effective. The primary mechanism of drug release from hydrophilic matrices occurs when the polymer swells on contact with the aqueous medium to form a gel layer on the surface of the system. The drug then releases by dissolution, diffusion and/or erosion [10-12].

Vast data were collected over the period of last 20 y to review the progressive developments in the field of matrix tablet research.

\section{Advantages of oral matrix systems}

This type of drug delivery has many benefits over conventional dosage forms, some of which are as follows [13-14]:

\section{Easy to manufacture.}

2. The frequency of application of dose is reduced, since the drug is released over a longer period of time. This is important to the patients with chronic illnesses and need a plasma drug concentrations o within its therapeutic range, such as, overnight management of pain in terminally ill patients.

3. 'Dose dumping' and toxic effects due to high plasma concentration are reduced.

4. Improvement in patient compliance.

5. Better control of therapeutic drug concentration.

6. Improvement in bioavailability of some drugs.

7. Increase the stability by protecting the drug from hydrolysis or other derivative changes in the gastrointestinal tract.

8. Cost-effective manufacturing is possible, since the number of doses are decreased.

Disadvantages of oral controlled release formulations

Like other formulations, it also possesses several disadvantages. These include [15-16]:

1. Expensive equipment and inert ingredients are required for some formulations. 
2. The drug release rate can be altered by food and gastric transit time; as a result, differences may arise in the release rate between doses.

3. If the formulations are crushed or chewed, it can lose the 'slow release' characteristics and possess toxicity.

4. A direct correlation of in vitro data with in vivo release is not possible without thorough and careful analysis. For example, there is a difference in the availability of water in different parts of the gastrointestinal tract and such factors need to be considered when designing tablets for extended release.

5. The dissolution characteristics should allow for the drug to be released in a controlled manner, highlighting the importance for the correct selection of polymers according to their physical, mechanical and pharmacokinetic properties.

\section{Rationale of developing sustained release matrix devices}

1. To extend the duration of action of the drug.

2. To reduce the frequency of dosing.

3. To reduce inter and intrasubject variability.

4. To minimize the fluctuations in plasma level.

5. To improve drug utilization.

6. To reduce adverse effects.

\section{Polymers used in matrix tablets}

There are number of polymers which may be used to formulate matrix tablets depending on the physicochemical properties of the drug substance to be incorporated into matrix system and drug release profile required. Polymers used for matrix tablets may be classified as [17]:

\section{1) Hydrogels:}

(a) Poly-hydroxyethyl methacrylate (PHEMA).

(b) Cross-linked polyvinyl alcohol (PVA).

(c) Cross-linked polyvinylpyrrolidone (PVP).

(d) Polyethylene oxide (PEO).

(e) Polyacrylamide (PA).

\section{2) Soluble polymers:}

(a) Polyethylene glycol (PEG).

(b) Polyvinyl alcohol (PVA).

(c) Polyvinylpyrrolidone (PVP).

(d) Hydroxypropyl methylcellulose (HPMC).

\section{3) Biodegradable polymers:}

(a) Polylactic acid (PLA).

(b) Polyglycolic acid (PGA).

(c) Polycaprolactone (PCL).

(d) Polyanhydrides.

(e) Polyorthoesters.

\section{4) Non-biodegradable polymers:}

(a) Polyethylene vinyl acetate (PVA).

(b) Polydimethylsiloxane (PDS).

(c) Polyether urethane (PEU).

(d) Polyvinyl chloride (PVC).

(e) Cellulose acetate (CA).

(f) Ethyl cellulose (EC).

\section{5) Mucoadhesive polymers:}
(a) Polycarbophil.
(b) Sodium Carboxymethylcellulose.
(c) Polyacrylic acid.
(d) Tragacanth.
(e) Methylcellulose.
(f) Pectin.

\section{6) Natural gums:}

(a) Xanthan gum.

(b) Guar gum.

(c) Karaya gum.

(d) Gum Arabic.

(e) Locust bean gum.

\section{Types of matrix systems}

The matrix system can be divided into five categories depending on the types of retarding agents or polymeric materials [17].

(1) Hydrophobic matrix system.

(2) Hydrophilic matrix system.

(3) Fat-wax matrix system.

(4) Biodegradable matrix

(5) Mineral matrix

\section{(1) Hydrophobic matrix systems}

As the term suggests, the primary rate-controlling components of the hydrophobic matrix are water-insoluble in nature. These ingredients include waxes, glycerides, fatty acids, and polymeric materials such as ethyl cellulose, Methyl Cellulose and acrylate copolymers. To alter drug release, it may be necessary to incorporate soluble ingredients such as lactose into the formulation. The presence of an insoluble ingredient in the formulations helps to maintain the physical dimension of the hydrophobic matrix during drug release. As such, diffusion of the active ingredient from the system is the release mechanism, and the corresponding release characteristic can be described by Higuchi kinetic model. In addition, hydrophobic matrix systems providing programmable rates of delivery have become more important. Constant rate delivery always has been one of the primary targets of controlled release system especially for a drug with narrow therapeutic index.

\section{(2) Hydrophilic matrix system}

The primary rate-limiting ingredients of the hydrophilic matrix are polymers that would swell when in contact with the aqueous solution and form a gel layer on the surface of the system. When the release medium (i.e. water) is thermodynamically compatible with a polymer, the solvent penetrates into the free spaces between macromolecular chains. The polymer may undergo a relaxation process, due to the stress of the penetrated solvent, so that the polymer chains become more flexible and the matrix swells. This allows the encapsulated drug to diffuse more rapidly out of the matrix. On the other hand, it would take more time for the drug to diffuse out of the matrix, since matrix swelling lengthens the diffusion path. It has been widely known that swelling and diffusion are not the only factors that determine the rate of drug release. For dissolvable polymer matrix, polymer dissolution is another important mechanism that can modulate the drug delivery rate. While either swelling or dissolution can be the predominant factor for a specific type of polymers, in most cases drug release kinetics is a result of a combination of these two mechanisms. The presence of water decreases the glassy-rubbery temperature (for HPMC from $184{ }^{\circ} \mathrm{C}$ to below $37^{\circ} \mathrm{C}$ ), giving rise to the transformation of glassy polymer to rubbery phase (gel layer). The enhanced mobility of the polymeric chain favors the transport of dissolved drug. Polymer 
relaxation phenomena determine the swelling or volume increase of the matrix. Depending on the polymer characteristics, the polymer amount in the rubbery phase, at the surface of the matrix, could reach the disentanglement concentration; the gel layer varies in thickness and the matrix dissolves or erodes. The concentration at which polymeric chains can be considered disentangled was demonstrated to correspond to an abrupt change in the rheological properties of the gel. It showed a relationship between the rheological behaviour of HPMC gels and their erosion rate, confirming that the polymer-polymer and polymer water interactions are responsible for the gel network structure and its sensitivity to erosion. In turn, they affect drug release rate in the case of poorly soluble drugs. The main polymers used in hydrophilic matrices are Hydroxyl propyl methyl cellulose (HPMC) and Hydroxyl propyl cellulose (HPC), Xanthan gum, Carbopol 940 and Alginates.
Hydrogel polymers were much investigated in the literature on the basis of drug release and release mechanism from hydrophilic matrix tablets as well as pellets. Hydroxyl propyl methyl cellulose (HPMC) and hydroxyl propyl cellulose (HPC) polymers achieve considerable attention due to their unique properties, and they can display good compression characteristics, including when directly compressed. They are nontoxic and can accommodate the high level of drug loading, and also having adequate swelling properties that allows rapid formation of an external gel layer, which retards or plays a major role in controlling drug release. Furthermore, HPMC polymers are well known as pH-independent materials, this advantage enables them to the drug is entrapped in the glassyrubbery core in the dry state. It forms a gelatinous layer upon hydration. However, this gelatinous layer is significantly different structurally from the traditional matrix tablets.

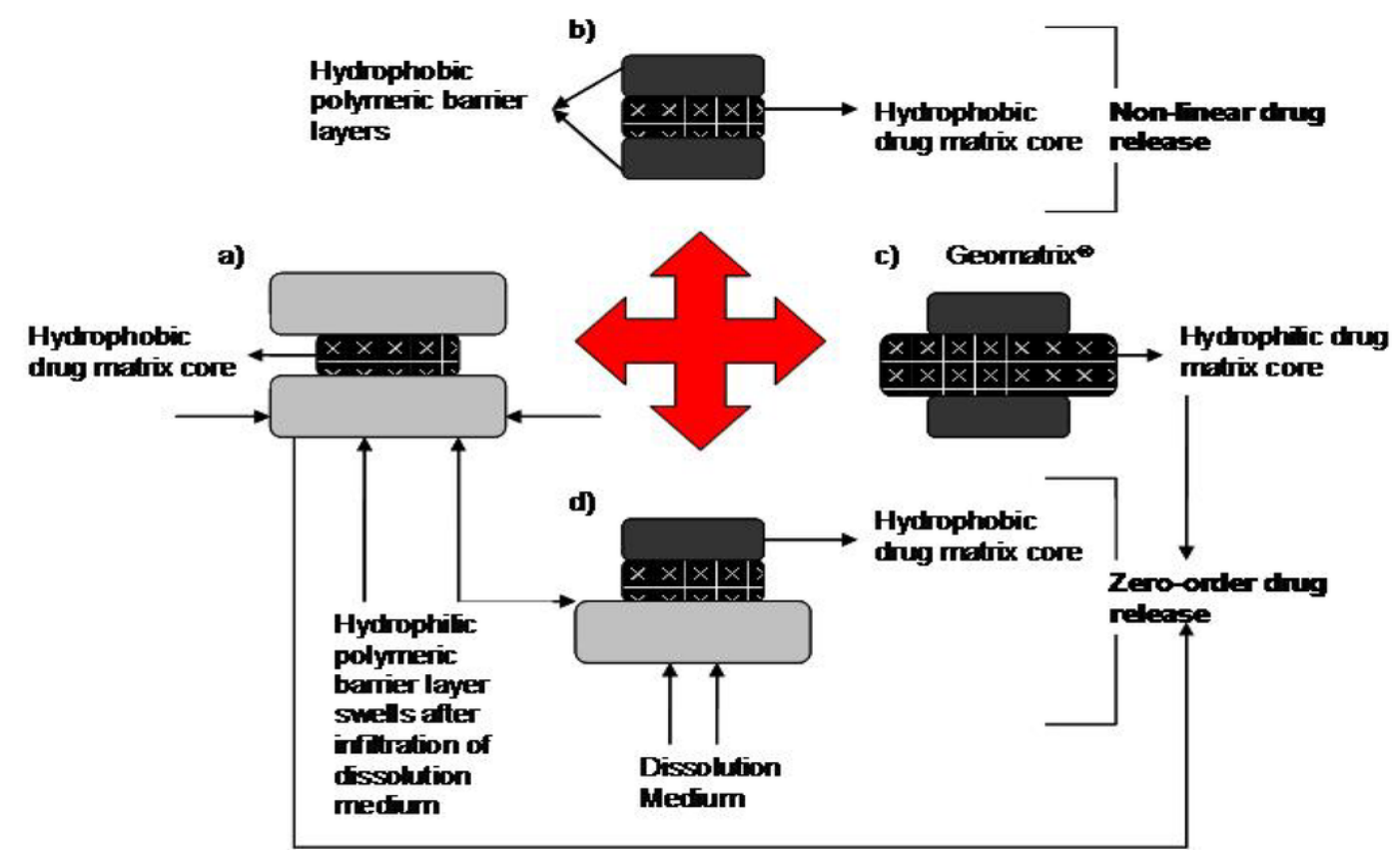

Fig. 1: Possible drug release mechanism from various matrix systems [18]

\section{(3) Fat-wax matrix systems}

The drug can be incorporated into fat-wax granulations by spray congealing in the air, blend congealing in an aqueous media with or without the aid of a surfactant and spray-drying techniques. In the bulk congealing method, a suspension of drug and melted fat-wax is allowed to solidify and is then comminuted for sustained release granulations. The mixture of active ingredients, waxy materials and fillers also can be converted into granules by compacting with roller compactor, heating in a suitable mixture such as fluidized-bed and a steam jacketed blender or granulating with a solution of waxy Material or other binders. The drug embedded into a melt of fats and waxes is released by leaching and/or hydrolysis as well as the dissolution of fats under the influence of enzymes and $\mathrm{pH}$ change in the gastrointestinal tract. The addition of surfactants to the formulation can also influence both the drug release rate and the proportion of total drug that can being corporate into a matrix [19].

\section{(4) Biodegradable matrix systems}

Biodegradable matrices are composed of monomers linked to one another through functional groups with unstable linkage. They degraded by enzymes generated by surrounding living cells or by nonenzymatic process into oligomers and monomers in the biological systems. Therese oligomers and monomers are then metabolized or excreted.

Examples are natural polymers such as proteins and polysaccharides; modified natural polymers; synthetic polymers such as aliphatic poly (esters) and poly anhydrides [20].

\section{(5) Mineral matrices}

The polymers obtained from different species of seaweeds are used to prepare mineral matrices. Alginic acid, a hydrophilic carbohydrate obtained from brown seaweeds (Phaephyceae) by the use of dilute alkali. On the basis of porosity of matrixthese are classified as (a) Macro porous; (b) Microporous and (c) Non-porous systems.

In macro porous systems, the diffusion of drug occurs through pores of the matrix, which are of size range 0.1 to $1 \mu \mathrm{m}$. In microporous system, the diffusion occurs essentially through pores but the pore size ranges between 50-200 $\AA$. In non-porous system no pores are found and the molecules diffuse through the network meshes [21].

\section{Different factors effecting rate drug release from matrix} systems

The release of drug from polymer matrix system is dependent upon the physicochemical properties of both drug and polymer as well as it is also dependent on several biological parameters [21].

\section{Physicochemical factors}

(1) Swelling property of polymer: Polymer dissolution includes absorption/adsorption of water in more accessible places, rupture of polymer-polymer linking with the simultaneous forming of waterpolymer linking, separation of polymeric chains, swelling and finally dispersion of polymeric chain in dissolution medium. Therefore, study of polymer hydration/swelling process for the polymers is required. 
(2) Drug solubility: Molecular size and water solubility of the drug are determinants in the release of drug from swelling and erosion controlled polymeric matrices. For drugs with reasonable aqueous solubility, the release of drugs occurs by dissolution infiltration medium and for drugs with poor solubility release occurs by both dissolution of drug and dissolution of drug particles through erosion of the matrix tablet.

(3) Solubility: In view of in vivo sink condition maintained actively by hem perfusion, it is logical that all the in vitro drug release studies should also be conducted under perfect sink condition.

(4) Polymer diffusivity: The diffusion of small molecules in polymer structure is energy activated process in which the diffusing molecules move to a successive series of equilibrium position when a sufficient amount of energy of activation for diffusion $E_{d}$ has been acquired by the diffusing molecules. It is dependent on length of polymer chain segment, cross linking and crystalline nature of polymers. The release of the drug may be attributed to the three factors such as polymer particle size, polymer viscosity and polymer concentration.

(5) Thickness of polymer diffusional path: The controlled release of a drug from both capsule and matrix type polymeric drug delivery system is essentially governed by Fick's law of diffusion: J D = D $\mathrm{dc} / \mathrm{dx}$ Where, J D is flux of diffusion across a plane surface of unit area D is diffusibility of drug molecule, $\mathrm{dc} / \mathrm{dx}$ is concentration gradient of drug molecule across a diffusion path with thickness $\mathrm{dx}$.

(6) The thickness of hydrodynamic diffusion layer: The magnitude of drug release value decreases on increasing the thickness of hydrodynamic diffusion layer $\delta \mathrm{d}$.

(7) Drug loading dose: The effect of initial drug loading of the tablets on the resulting release kinetics is more complex in case of poorly water-soluble drugs, with increasing initial drug loading the relative release rate first decreases and then increases, whereas, absolute release rate monotonically increases. When the amount of drug present at a certain position within the matrix, exceeds the amount of drug soluble under given conditions, the excess of the drug has to be considered as non-dissolved and thus not available for diffusion.

(8) Diluent's effect: The Water soluble diluents like lactose cause marked an increase in drug release rate and release mechanism is also shifted towards Fickian diffusion; while insoluble diluents like dicalcium phosphate reduce the Fickian diffusion and increase the relaxation (erosion) rate of the matrix. The reason behind this is that water-soluble filler in matrices stimulates the water penetration into inner part of matrix, due to increase in hydrophilicity of the system, causing rapid diffusion of the drug, leads to increased drug release rate.

(9) Additives: The effect of adding non-polymeric excipients to a polymeric matrix has been claimed to produce an increase in the release rate of hydrosoluble active principles.

(10) Dose size: For orally administered systems, there is an upper limit to the bulk size of the dose to be administered. Compounds that require large dosing size can sometimes be given in multiple amounts or formulated into liquid systems. Another consideration is the margin of safety involved in the administration of a large amount of a drug with a narrow therapeutic range.

(11) Ionization, pka and aqueous solubility: Most drugs are weak acids or bases. Since the unchanged form of a drug preferentially permeates across lipid membranes, it is important to note the relationship between the pka of the compound and the absorptive environment. Delivery systems that are dependent on diffusion or dissolution will likewise be dependent on the solubility of the drug in aqueous media. These dosage forms must function in an environment of changing $\mathrm{pH}$, the stomach being acidic and the small intestine more neutral, the effect of Phone the release process must be defined. Compounds with very low solubility $(<0.01 \mathrm{mg} / \mathrm{ml})$ are inherently sustained, since their release over the time course of a dosage form in the GI tract will be limited by dissolution of the drug.

(12) Partition coefficient: It is common to consider that the biological membranes are lipidic; therefore the partition coefficient of oil-soluble drugs becomes important in determining the effectiveness of membrane barrier penetration. Compounds which are lipophilic in nature having high partition coefficient are poorly aqueous. Sustained release drug delivery system is not required to retain in the lipophilic tissue for the longer time. In case of compounds with low partition coefficient, it is difficult for them to penetrate the membrane, resulting in poor bioavailability. The choice of diffusion-limiting membranes must largely depend on the partitioning characteristics of the drug.

(13) Stability: Orally administered drugs can be subject to both acid-base hydrolysis and enzymatic degradation. For the dosage form that are unstable in the stomach, systems that prolong delivery over the entire course of transit in the GI tract are beneficial; this is also true for systems that delay the release until the dosage form reaches the small intestine. Compounds that are unstable in the small intestine may demonstrate decreased bioavailability when administered from a sustaining dosage form. This is because more drugs is delivered in the small intestine and, hence, is subject to degradation. Propantheline and probanthine are representative examples of such drug.

\section{Biological factors}

(1) Biological half-life: Every drug has its own characteristic elimination rate, which is the sum of all elimination processes, including metabolism, urinary excretion and all over processes that permanently remove the drug from the bloodstream. Therapeutic compounds with a short half-life are generally are an excellent candidate for sustained release formulation, as this reduces dosing frequency. Normally, drugs with a half-life shorter than $2 \mathrm{~h}$ such as furosemide or levodopa are poor candidates for this type of preparation.

(2) Absorption: If the transit time of any drug in the absorptive areas of the GI tract is about 8-12 h, the maximum half-life for absorption should be approximately 3-4 h; otherwise, the device will pass out of the potential absorptive regions before drug release is complete. One method to provide sustaining mechanisms of delivery for compounds tries to maintain them within the stomach. This allows slow release of the drug, which then travels to the absorptive site. Other attempt is to formulate low-density pellet or capsule. Another approach is that of bioadhesive materials.

(3) Metabolism: Drugs those are significantly metabolized before absorption, either in the lumen or the tissue of the intestine, can show decreased bioavailability from the slower-releasing dosage form.

Drug release kinetics from sustained release matrix system

\section{Zero-order kinetics}

A zero order release would be predicted by the following equation:

$\mathrm{Qt}-\mathrm{Q}_{0}=\mathrm{K}_{0} \mathrm{t}$

Where, $Q_{t}=$ Amount of drug release dissolved in time ' $t$ '.

$Q_{0}=$ Initial amount of drug concentration in solution.

$\mathrm{K}_{0} \mathrm{t}=$ Zero order rate constant.

When the data are plotted as cumulative percent drug release versus time, if the plot is linear then data obeys zero order kinetics with slope $\mathrm{K}_{0}$. This model represents an ideal release profile to achieve the prolonged pharmacological action.

\section{First order kinetics}

A first order release would be predicted by the following equation:

$\log Q t=\log Q o-K_{1} t / 2.303 \ldots$ Where, $Q_{t}=$ Amount of drug released in time' $t$ '. Qo = Initial amount of drug concentration in solution. $K_{1} t=$ First order rate constant. When data were plotted as log cumulative percent drug remaining verses time yields a straight line indicating that the release follows first order kinetics. The constant release rate, $\mathrm{K}$ can be obtained multiplying slope values.

\section{Higuchi's model}

Drug release from the matrix device by diffusion has been described by Higuchi's equation: 
$Q=\sqrt{D} \delta / \tau(2 C-\delta C s) C s t \ldots$ Where, $Q=$ Amount of drug released in time ' $t$ ', $\mathrm{D}=$ Diffusion coefficient of the drug in the matrix. Cs = Solubility of the drug in the matrix. $\delta=$ Porosity of matrix. $\tau=$ Tortuosity. $t=$ Time (h).

The equation may be simplified then the equation becomes; $Q=K_{H}$ $x \mathrm{t}^{1 / 2}$ Where, $\mathrm{K}_{\mathrm{H}}=$ Higuchi dissolution constant.

When data are plotted according to this equation, i.e., cumulative drug released verses square root of time, yields a straight line, indicating that the drug was released by diffusion controlled mechanism [22].

\section{Drugs suitable to be formulated as matrix tablets}

Drugs with a low half-life $(<5$ h.), freely soluble in water and larger therapeutic window can be formulated as sustained release matrix systems. The drugs with suitable polymer and combination of polymers to prepare matrix are enlisted in table 1.

Table 1: Combination of few drugs and polymers formulated into matrix tablets

\begin{tabular}{|c|c|c|c|}
\hline Drug & Polymers & Drug & Polymers \\
\hline Zidovudine & HPMC-K4M, Carbopol-934, EC & Furosemide & Guar gum, Pectin, Xanthan gum \\
\hline Venlafexine & Beeswax, Caranuaba wax & Acarbose & HPMC, Eudragit \\
\hline Domperidone & HPMC-K4M, Carbopol-934 & Aceclofenac & HPMC-K4M,K15M, K100M,E15,EC, Guar gum \\
\hline Alfuzosin & HPMC-K15M, Eudragit-RSPO & Ambroxol HCL & HPMC-K100M, \\
\hline Minocycline & HPMC-K4M, HPMC-K15M, EC & Aspirin & EC, Eudragit-RS100, S100 \\
\hline Ibuprofen & EC, CAP & Diclofenac $\mathrm{Na}$ & Chitoson, EC, HPMCP, HPMC \\
\hline Metformin HCL & HPMC-K100M, EC & Diethylcarbamazine citrate & Guar gum, HPMC-E15LV \\
\hline Propranolol HCL & Locust bean gum, HPMC & Diltiazem & $\begin{array}{l}\text { HPMC-K100M, HPMC-K4M, Karaya gum, Locust } \\
\text { bean gum, Sod. CMC }\end{array}$ \\
\hline Enalpril meleate & HPMC-K100M,HPMC K4M, & Miconazole & Pectin, HPMC \\
\hline Flutamide & $\begin{array}{l}\text { HPMC-K4M, Sod. CMC, Guar gum, } \\
\text { Xanthan gum }\end{array}$ & Naproxen & HPMC-K100M, HPMC-K15M, PVP \\
\hline Indomethacin & EC, HPMC & Nicorandil & HPMC, CMC, EC \\
\hline $\begin{array}{l}\text { Chlorpheniramine } \\
\text { meleate }\end{array}$ & Xanthan gum, Chitoson & Ondansertan & HPMC-K100M, HPMC-K4M, HPMC-K15M \\
\hline Itopride HCL & HPMC-K100M, HPMC-K4M, EC & Phenytoin $\mathrm{Na}$ & Tragacanth, Acacia, Guar gum, Xanthan gum \\
\hline Losartan potassium & $\begin{array}{l}\text { HPMC-K100M, HPMC-K4M, } \\
\text { Eudragit-RSPO }\end{array}$ & Ranitidine HCL & Chitoson, Carbopol-940 \\
\hline Metoclopromide & HPMC, CMC, EC, SSG & Theophylline & $\begin{array}{l}\text { Carbopol-934P, HPMC-K100M, HPMC-K4M, HPMC- } \\
\text { K15M, EC }\end{array}$ \\
\hline Tramadol & HPMC-K4M, Karaya gum, & Amlodipine & HPMC, EC \\
\hline
\end{tabular}

\section{CONCLUSION}

From the discussion, it can be concluded that matrix tablets, developed by using a rational combination of polymers can successfully applied to sustain the release of the drug. Sustaining the release of the drug may be helpful in increasing the efficiency of the drug as well as they are also useful to improve patient's compliance. The systems are economic since these are developed by using the commonly available polymers. These systems are especially useful in case of the patients who need a constant delivery of drug for a longer period of time.

\section{ACKNOWLEDGEMENT}

The author is grateful to the Principal and the authority of Pulla Reddy Institute of Pharmacy, Annaram (V), Jinnaram (M), Telengana, India for providing the necessary facilities to prepare this review article.

\section{AUTHOR CONTRIBUTION}

All the work have carried out by me.

\section{CONFLICT OF INTERESTS}

Author confirms that there are no conflicts of interest

\section{REFERENCES}

1. Kumar S, Kumar A, Gupta V, Malodia K, Rakha P. Oral extended release drug delivery system: a promising approach. Asian J Pharm Technol 2012;2:38-43.

2. Yang Lina S, Kawashimab Y. Current status and approaches to developing press-coated chronodelivery drug systems. J Controlled Release 2012;157:331-53.

3. Sharma A, Sharma S, Jha KK. The study of salbutamol matrix tablets using different polymers as release retarding agent. Pharma Res 2009;1:15-22.

4. Mandal UK, Chatterjee B, Senjoti FG. Gastro-retentive drug delivery systems and their in vivo success: a recent update. Asian J Pharm Sci 2016;11:575-84.
5. Das MK, Sahu BP, Hazarika JNR. Development of bilayer tablets for the immediate and controlled release of allicin. Int J Curr Pharm Res 2017;9:153-60.

6. Ain S, Kumar B, Pathak K. Development and characterization of controlled release famotidine matrix tablets containing complexes. Int J Appl Pharm 2017;9:38-46.

7. Rajput GC, Majmudar FD, Patel JK, Thakor RS, Patel BP, Rajgor NB. Stomach-specific mucoadhesive microsphere as a controlled drug delivery system. Int J Pharm Biomed Res 2010;1:30-41.

8. Olivares-Morales A, Kamiyama Y, Darwich AS, Aarons L, Rostami-Hodjegan A. Analysis of the impact of controlled release formulations on oral drug absorption, gut wall metabolism and relative bioavailability of CYP3A substrates using a physiologically-based pharmacokinetic model. Eur J Pharm Sci 2015;67:32-44.

9. Shin KH, Yoon G, Yoon IS, Park JW. Preparation and evaluation of oral controlled-release cilostazol formulation: pharmacokinetics and antithrombotic efficacy in dogs and healthy male Korean participants. J Pharm Pharmacol 2014;66:961-74.

10. Neeharika MS, Jyothi BJ. Chronotherapeutics: an optimizing approach to synchronize drug delivery with circadian rhythm. J Crit Rev 2015;2:31-40.

11. Kubova K, Pecek D, Hasserova K, Dolezel P, Pavelkova M, Vyslouzil J, et al. The influence of thermal treatment and type of insoluble poly (meth)acrylates on dissolution behavior of very soluble drug from hypromellose matrix tablets evaluated by multivariate data analysis. Pharm Dev Technol 2017;22:206-17.

12. Jayanthi B, Manna PK, Madhusudhan S, Mohanta GP, Manavalan R. Per oral extended release products-an overview. J Appl Pharm Sci 2011;1:50-5.

13. Li L, Zhang X, Gu X, Mao S. Applications of natural polymeric materials in solid oral modified-release dosage forms. Curr Pharm Des 2015;21:5854-67. 
14. Agüero L, Zaldivar-Silva D, Peña L, Dias ML. Alginate microparticles as oral colon drug delivery device: a review. Carbohydr Polym 2017;168:32-43.

15. Semjonov K, Kogermann K, Laidmäe I, Antikainen O, Strachan CJ, Ehlers $\mathrm{H}$, et al. The formation and physical stability of twophase solid dispersion systems of indomethacin in supercooled molten mixtures with different matrix formers. Eur J Pharm Sci 2017;97:237-46.

16. Zimmer $七$, Kasperek R, Poleszak E. Modern polymers in matrix tablets technology. Polim Med 2014;44:189-96.

17. Chidambaram N, Porter W, Flood K, Qui Y. Formulation and characterisation of new layered diffusional matrices for zeroorder sustained release. J Controlled Release 1998;52:14958.

18. Zou M, Wang Y, Xu C, Cheng G, Ren J, Wu G. Wax-matrix tablet for time-dependent colon-specific delivery system of sophora flavescens aiton: preparation and in vivo evaluation. Drug Dev Ind Pharm 2009;35:224-33.

19. Campiñez MD, Aguilar-de-Leyva A, Ferris C, de Paz MV, Galbis JA, Caraballo I. Study of the properties of the new biodegradable polyurethane PU (TEG-HMDI) as matrix forming excipient for controlled drug delivery. Drug Dev Ind Pharm 2013;39:1758-64.

20. Kanjanabat S, Pongjanyakul T. Preparation and characterization of nicotine-magnesium aluminum silicate complex-loaded sodium alginate matrix tablets for buccal delivery. AAPS PharmSciTech 2011;12:683-92.

21. Krajacic $A B$, Tucker G. Matrix formation in sustained release tablets: possible mechanism of dose dumping. Int J Pharm 2003;251:67-78.

22. Shaikh HK, Kshirsagar RV, Patil SG. Mathematical models for drug release characterization: a review. World J Pharm Res 2015;4:324-38. 